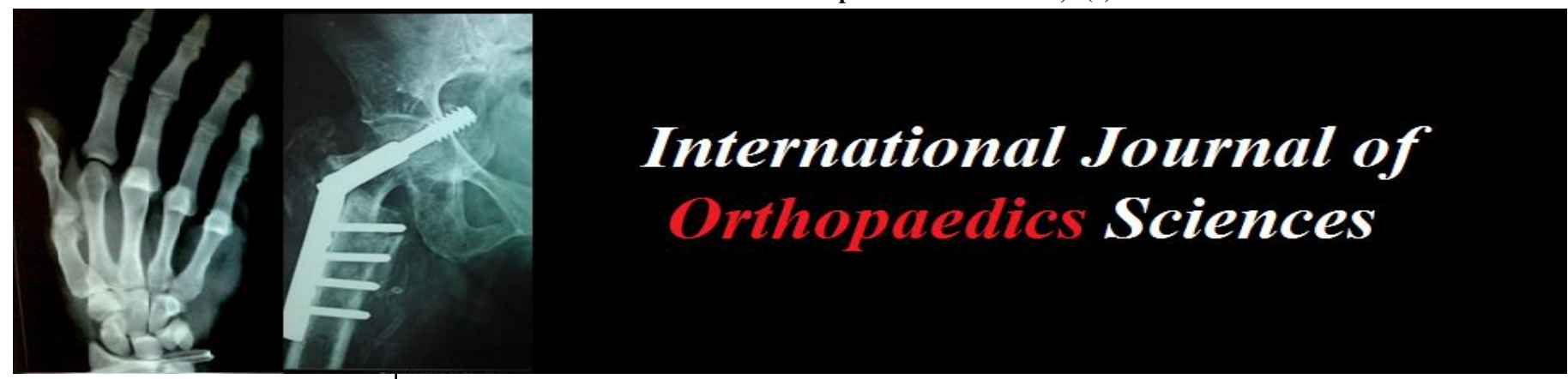

E-ISSN: 2395-1958

P-ISSN: 2706-6630

IJOS 2021; 7(3): 592-596

(C) 2021 IJOS

www.orthopaper.com

Received: 13-05-2021

Accepted: 15-06-2021

Dr. Sreenivasulu PSB

Assistant Professor, Narayana

Medical College and Hospital

Nellore, Andhra Pradesh, India

\section{Dr. Aleem}

Post Graduate, Narayana

Medical College and Hospital

Chintareddypalem, Nellore,

Andhra Pradesh, India

\section{Dr. Harikrishna Reddy}

Associate Professor, Narayana

Medical College and Hospital,

Chintareddypalem, Nellore,

Andhra Pradesh. India
Corresponding Author: Dr. Sreenivasulu PSB

Assistant Professor, Narayana

Medical College and Hospital

Nellore, Andhra Pradesh, India

\section{A comparative study of functional outcome of proximal femoral nailing and bipolar hemiarthroplasty in unstable basicervical fracture neck of femur in age $>45$ years}

\section{Dr. Sreenivasulu PSB, Dr. Aleem and Dr. Harikrishna Reddy}

DOI: https://doi.org/10.22271/ortho.2021.v7.i3i.2807

\section{Abstract}

Background: A basicervical femoral neck fracture, which is located at the junction between the femoral neck and intertrochanteric region, is a rare type of fracture. (1) Despite several randomized trials and comparative studies treatment of unstable basicervical fractures in elderly patients is still controversial, which we want to investigate.

Methods: A prospective cohort analysis of 44 patients, of which 22 each were operated with cemented bipolar hemiarthroplasty (BPA) and proximal femur nail (PFN) between January 2018 till July 2021 was done. Primary outcome was assessed in both groups using Harris hip score( HHS) at 4weeks, 6 weeks, 12 weeks, 6months and 1 year on outpatient basis. The secondary outcome measures were intraoperative blood loss, surgical time, functional outcome, range of motion limb length discrepancy at final follow up. All complications were noted at follow up.

Results: The HHS was significantly higher for BPA group at all follow ups, at 4 weeks of follow up was 79.81 for BPA and 73.18 for PFN (p-value $=0.001$ ) and at 1 year of follow up was 88.55 for BPA and 79.03 for PFN (p-value $=0.001$ ). Statistically significant differences were found between BPA and PFN groups with reference to intraoperative blood loss, surgical time, functional outcome, range of motion, limb length discrepancy at final follow up.

Conclusion: The bipolar hemiarthroplasty group had fewer complications and earlier mobilization with better Harris hip score at all follow ups, which indicate bipolar hemiarthroplasty is a better option in the treatment of unstable basicervical fractures in elderly patients.

Keywords: unstable basicervical fractures, bipolar hemiarthroplasty (BPA), proximal femur nailing (PFN), harris hip score (HHS)

\section{Introduction}

Fracture neck of femur (intracapsular) accounts for more than 60 percent of fractures of proximal femur.

The incidence hip fractures is approximately 80 per 100,000 persons and is expected to double over the next 50 years as the population ages. Intracapsular and intertrochanteric fractures makes up $85 \%$ of these fractures. It is estimated that the number of these fractures is 1.26 million cases annually worldwide, which is expected to amount to 2.6 million cases in 2025 and to 4.5 million cases in 2050. There is, in fact, an increase in the incidence of these fractures in correlation with the aging of the population, especially at old age and in female populations over 60 and are doubled with a 10-year increase in age.

Due to problems caused by these fractures and an increase in the number of the elderly population leading to a significant increase in the incidence of these fractures, it is absolutely necessary to use an effective and appropriate treatment modality for such patients. Many treatment methods have been used for the reduction of intracapsular and intertrochanteric fractures, including dynamic hip screw (DHS), trochanteric buttressing plate, proximal femoral nail (PFN), unipolar and bipolar hemiarthroplasty and external fixation.

However, it is difficult to achieve and maintain a stable fixation in elderly patients due to their poor bone quality The understanding of fracture stability is paramount in decision making and 
the choice of implant Unstable basicervical fractures in the elderly pose certain special problems. In this age group the fracture configuration is generally comminuted with presence of extensive osteoporosis. There is problem with correct and accurate placement of the implant and hold of the implant hence prolonged immobilization is required to achieve complete union. On the other hand there is a need for rapid full weight bearing mobilization of this group of patients as they are generally medically compromised due to age and associated diseases. In addition, these patients may not have adequate psychomotor skills required for graded and protected weight bearing required with internal fixation. Hence there are two conflicting requirements that need to be addressed to in a balanced way. So despite the publication of reports of randomized trials and comparative studies of treatment of unstable basicervical patients is still controversial. In these patients due to combination of osteoporosis and instability, early resumption to full weight bearing is difficult. Because of prolonged immobilization, complications like deep vein thrombosis, pressure sores, dehydration, metabolic disturbances, etc are likely. So they have increased the morbidity and mortality threshold.

Despite advances in internal fixation techniques, the failure rate of the dynamic hip screw is $7.6 \%$ to $10.8 \%$ while failure rate of the proximal femoral nail is between $7.1 \%$ and $14.5 \%$ in unstable fractures. There have been various reports of successful outcomes after the use of hemiarthroplasty and total hip arthroplasty ${ }^{[5]}$.

Hemiarthroplasty and THA, as two possible treatment options for unstable basicervical fractures, may offer the potential for quick recovery with little risk of mechanical failure, avoid the risks often associated with internal fixation, and enable patients to maintain a good level of function immediately after surgery. Treatment of unstable basicervical fracture is still controversial, despite of the publication of reports of randomized trials and comparative studies and their role in unstable osteoporotic and severely comminuted fractures is still to be defined ${ }^{[3]}$.

The purpose of this prospective study is to analyze and compare the role of primary bipolar hemiarthroplasty (BPA) and proximal femur nailing (PFN) in cases of unstable intracapsular femur fractures in elderly.

\section{Materials \& Methods}

From January 2018 till July 2021 a total of 44 patients with unstable basicervical fractures (AO/OTA 31A,31B3) were included in a prospective cohort study conducted at Narayana medical college and hospital, Nellore, Andhra Pradesh, India. The study protocol was approved by Institutional Ethics Committee.

A total of 44 patients, of which 22 each were operated with bipolar hemiarthroplasty and PFN were selected for the study. All surgeries were performed within an average period of 2 days for BPA and 3.92 days for PFN. The mean age in BPA group was 63 years and 56 years PFN group.

Of the 22 fractures in BPA group, 15 were $31 \mathrm{~B} 3$ and 7 were 31A2.3, where-as in PFN group out of 22 cases, 9 were 31B3, 9 were A2.2 and 4 were 31 A2.3. The patients were operated in lateral decubitus position for BPA and supine on a fracture table for PFN. Antibiotic prophylaxis was followed (1 gm Ceftriaxone IV 30 minutes before procedure) as hospital protocols. All the cases were operated by the two lead surgeons of the hospital. The patients were allowed to bear weight as tolerated in cases of stable reduction in PFN group and $1^{\text {st }}$ post-operative day for BPA.

After clinical and radiological examination the clinical and functional outcomes were assessed in both groups using Harris hip score (HHS) at 4 weeks, 6 weeks, 12weeks, 6 months and 1 year on outpatient basis.

We classified the quality of reduction in PFN group as anatomical $(<5$ degrees of varus, valgus, anteversion or retroversion), acceptable (5-10 degrees) or poor (>10 degrees). A stem was considered to be unstable when there was progressive subsidence exceeding $3 \mathrm{~mm}$, any change in position, or a continuous radiolucent line wider than $2 \mathrm{~mm}$ at the bone-cement interface. The fracture was considered to have healed when callus was visible and the patient did not feel any pain at the fracture site.

\section{Results}

The study included 22 patients in each group consisting 13 females and 9 males in BPA group whereas in PFN group there were 8 females and 14 males. In both groups mainly the mode of injury was self-fall. The patients in both the groups were comparable in their demographics as shown in table1.

The average blood loss was $300 \mathrm{ml}$ in BPA and $90 \mathrm{ml}$ in PFN which is statistically significant $(\mathrm{p}$-value $=0.001)$. The mean follow up of both the groups was 12 months. The time for surgery was shorter in PFN group (45 minutes) as compared to BPA (75 minutes) ( $\mathrm{p}$ value: 0.001). Mobilization was earlier in BPA group (3 days). The average full weight bearing time in PFN group was 5 weeks.

The Functional outcome assessment of both these procedures were evaluated using Harris Hip Score is shown in table 2. The mean HHS, at 4 weeks of follow up was 79.81 for BPA and 73.18 for PFN (p-value $=0.001$ ), at 6 weeks of follow up was 81.65 for BPA and 74.81 for PFN ( $\mathrm{p}$-value $=0.001$ ). The mean HHS at 12 weeks of follow up was 82.77 for BPA and 75.74 for PFN (p-value $=0.001$ ), at 6 months of follow up was 85.63 for BPA and 77.25 for PFN (p-value $=0.001$ ) and at 1 year of follow up was 88.55 for BPA and 79.03 for PFN $(p$-value $=0.001)$. The radiographic pictures with follow up details are depicted in figure 1,2 and 3.

We had complications related to surgery and prosthesis/implant in both groups. We had 2 superficial surgical site infections in BPA group and 1 patients had superficial surgical site infection in PFN group which were treated with appropriate antibiotics. In BPA group additionally 1 patient had deep infection, 1 patient had bed sores and 1 patient had DVT.

All these complications were not seen in PFN group

Two patients had implant related complications in PFN group like distal locking screw loosening which was treated appropriately. One patient had abductor weakness in PFN group. One patient in the PFN group had died due reasons other than related to the surgery. 
Table 1: Demographic, preoperative and postoperative data.

\begin{tabular}{|c|c|c|c|}
\hline Characteristics & Bipolar & PFN & P value \\
\hline Mean Age & 63 & 56 & 0.001 \\
\hline Sex (M:F) & $9: 13$ & $14: 8$ & 0.054 \\
\hline Mracture Type (31B3:A2.2:A2.3) & $15: 0: 7$ & $9: 9: 4$ & - \\
\hline Mean TTS & 2.2 & 3.8 & 0.001 \\
\hline Mean ST & 75 & 45.63 & 0.001 \\
\hline Mean Hospital Stay & 16 & 17.926 & 0.379 \\
\hline Mean LLD & 0.83 & 0.14 & 0.001 \\
\hline Mean BTOC & 1.33 & 3.13 & 0.001 \\
\hline Mean CTOW & 3 & 5.35 & 0.001 \\
\hline
\end{tabular}

M: Male; F: Female;R: Right; L: Left; TTS: Time To Surgery; ST: Surgery Time; LLD: Limb Length Discrepancy; BTOC: Bed To Chair; CTOW: Chair To Walking
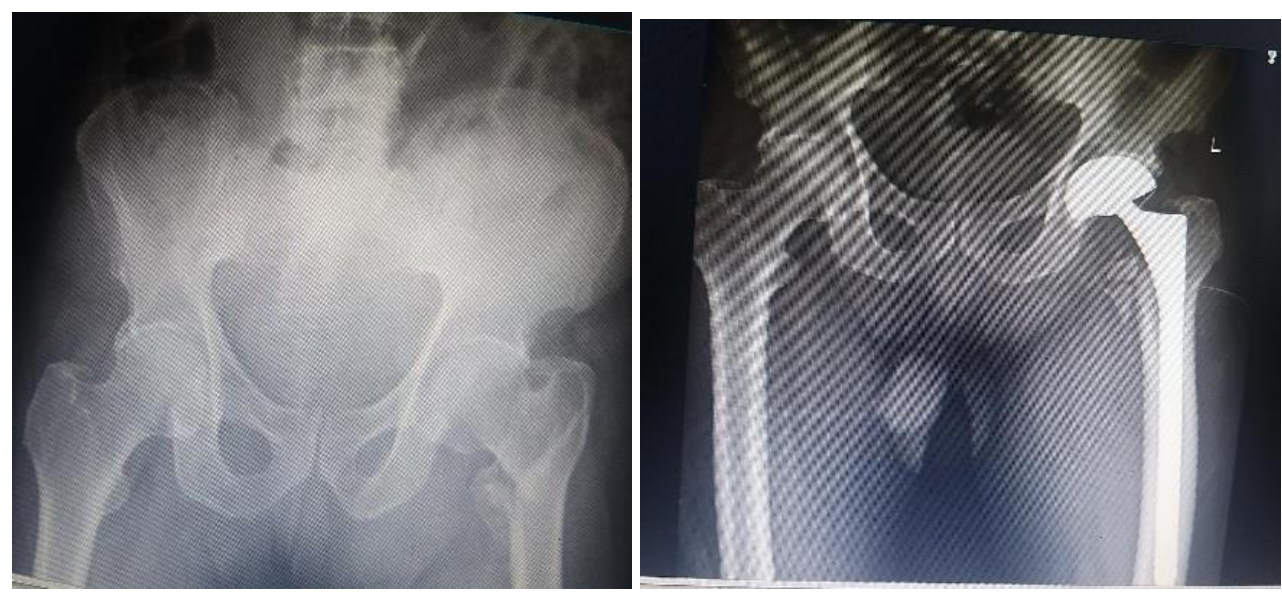

Fig 1: A: Pre-op radiographs IT fracture, B: Immediate post-op by cemented Bipolar hemiarthroplasty

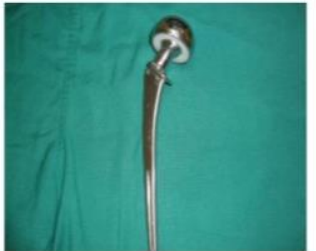

Bipolar prosthesis

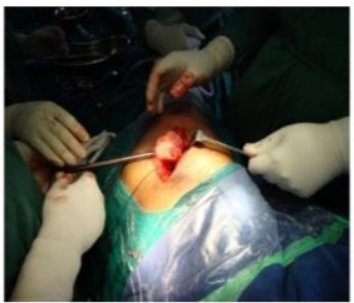

Femoral head delivered out

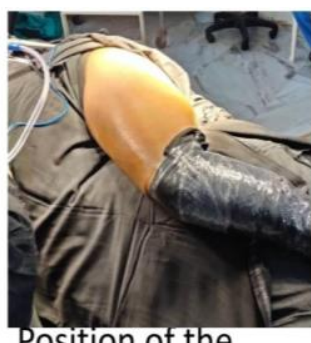

Position of the

patient
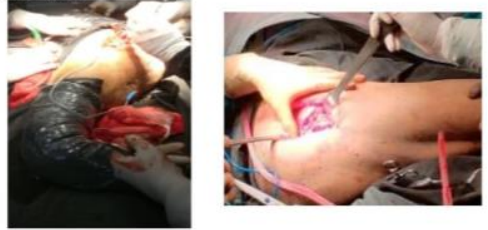

Preparation of stem
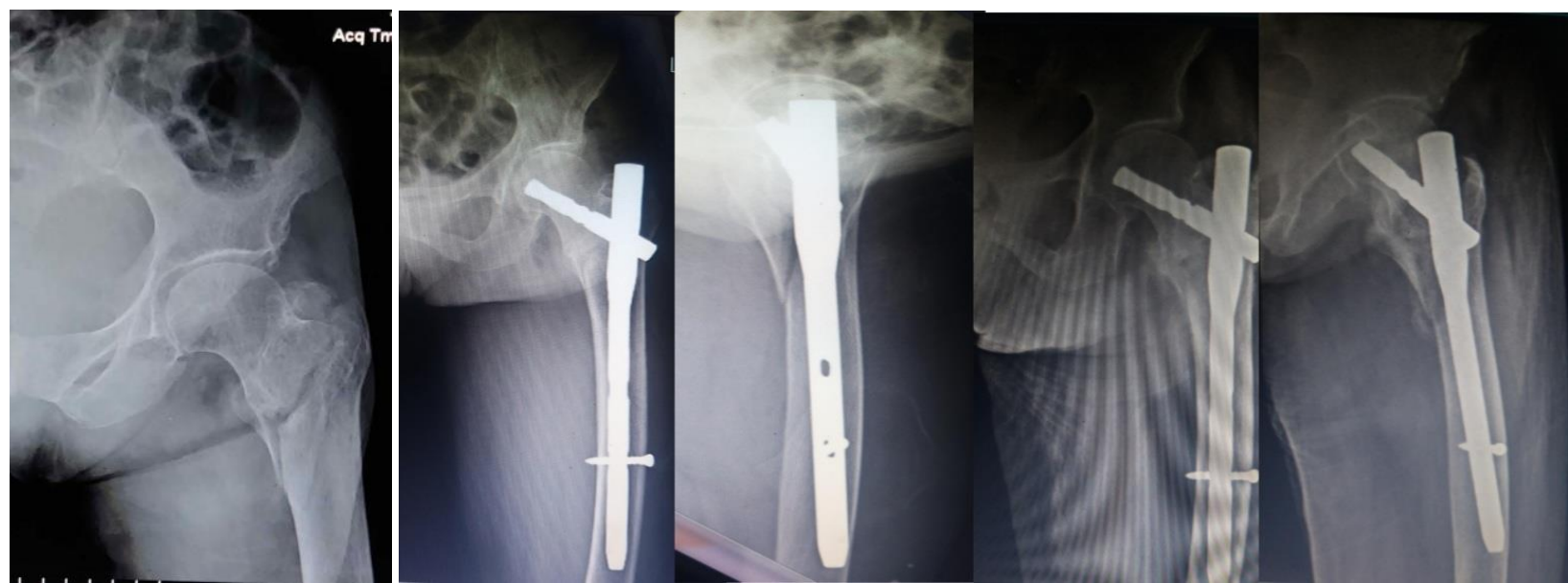

Fig 2: A: Pre-op radiographs IT fracture, B and C: Post-op anterio- posterior at POD 1, at 4 weeks, 3 months, 1year $\sim 594 \sim$ 

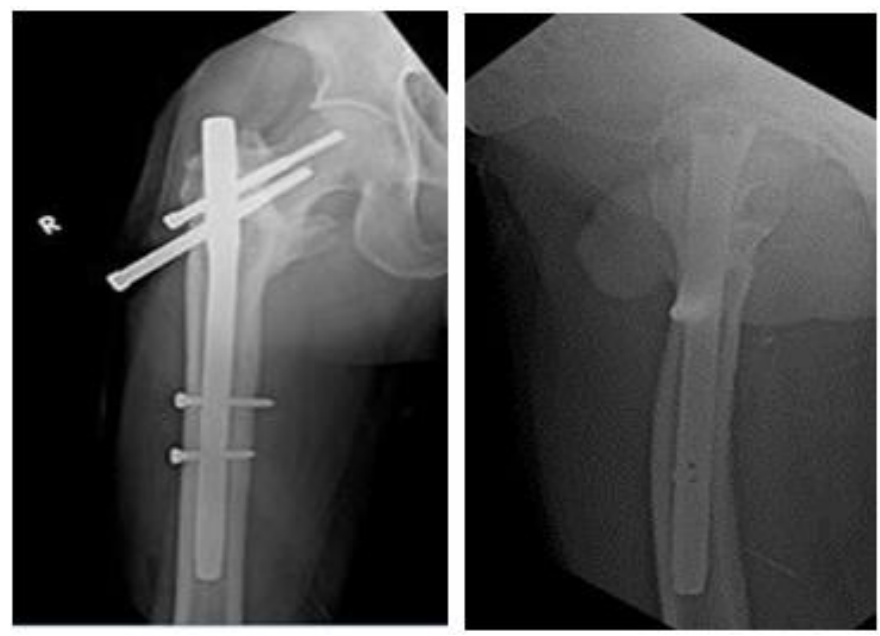

Fig 3: A and B: showing inferior lag screwback out (Z-effect) as a PFN complication.

Table 2: Functional outcome assessment using Harris Hip Score.

\begin{tabular}{|c|c|c|c|c|c|c|}
\hline & Group & No. & Mean & Std. Deviation & Std. Error Mean & P-value \\
\hline \multirow{2}{*}{ Post Op 4 Weeks } & $\mathrm{BPA}$ & 22 & 77.815 & 2.3540 & 0.4530 & \multirow[b]{2}{*}{0.001} \\
\hline & PFN & 22 & 71.185 & 3.6903 & 0.7102 & \\
\hline \multirow{2}{*}{ Post Op 6 Weeks } & BPA & 22 & 79.889 & 2.3912 & 0.4602 & \multirow[b]{2}{*}{0.001} \\
\hline & PFN & 22 & 72.815 & 3.9325 & 0.7568 & \\
\hline \multirow{2}{*}{ Post Op 12 Weeks } & BPA & 22 & 81.778 & 2.3588 & 0.4540 & \multirow[b]{2}{*}{0.001} \\
\hline & PFN & 22 & 74.741 & 4.1933 & 0.8070 & \\
\hline \multirow{2}{*}{ Post Op 6 Months } & $\mathrm{BPA}$ & 22 & 83.630 & 3.4211 & 0.6584 & \multirow[b]{2}{*}{0.001} \\
\hline & PFN & 22 & 75.259 & 4.3729 & 0.8416 & \\
\hline \multirow{2}{*}{ Post Op 1 Year } & $\mathrm{BPA}$ & 22 & 85.556 & 2.7363 & 0.5266 & \multirow[b]{2}{*}{0.001} \\
\hline & PFN & 22 & 77.037 & 4.1276 & 0.7944 & \\
\hline
\end{tabular}

\section{Discussion}

Our study included 44 patients, 22 in each group and patients were followed up till 12 months post operatively. We found that the mean blood loss during BPA surgery was $300 \mathrm{ml}$ and during PFN surgery the blood loss was $90 \mathrm{ml}$ which is significantly lower (p-value=0.001).

The mean limb length discrepancy in BPA group was significantly higher compared to PFN group.

In elderly patients, achieving and maintaining the reduction is a major problem during the fracture treatment. The implant related complications like cut through, screw back out are particularly more in this patient group. In our study we had one implant related complication. These complications are fewer in a cemented bipolar hemiarthroplasty. These implant related complications of PFN surgeries can be minimized by proper pre-operative planning and correct positioning of the screws.

The average duration of surgery in PFN group is shorter than BPA group considering the invasive nature of surgery of BPA group. Complications like surgical site infections including both superficial and deep infections were higher in the BPA group compared to the PFN group. These complications can also be attributed the duration of surgery and invasive nature of surgery. We had 2 superficial surgical site infection and 1 deep infection in BPA group which were treated appropriately as per the infection control guidelines. The superficial and deep infections in the PFN group in our study were 1 and 0 respectively.

The average bed to chair mobilization time in BPA group was 1.33 days compared to 3.13 days in PFN group. The average chair to walker time in BPA group was 3 days and in PFN group was 5.18 weeks. These findings show that mobilization is much shorter in BPA group at each level which reduces overall hospital stay and complications related to it.In our study 1 patients of BPA group had bed sores and one patient had DVT which were treated conservatively, unlike the PFN group where these complications were not seen. These findings are similar to previous studies where these complications were either less or not seen in PFN group.

The BPA group consistently had statistically significant higher Harris Hip Scores compared to PFN group at all the follow ups up to one year. Harris hip scores were 85.55 and 77.03 in BPA and PFN groups, respectively.

The results of our study showed that both BPA and PFN are good treatment methods with better post operative outcomes in case of BPA which was also reported in other studies The limitation of this study is a shorter follow up period and that all the surgeries were not performed by same surgeon. Hence further long term follow up studies are required to compare the function outcomes of these two different procedures. The surgeon should choose an ideal method as per the individual case requirements.

\section{Conclusion}

Patients undergoing bipolar hemiarthroplasty had an earlier mobilization than patients treated with proximal femur nailing, but the surgical procedure time was shorter in the patients undergoing fixation with proximal femur nailing. Bipolar hemiarthroplasty is a better option in the treatment of unstable basicervical fractures in elderly patients

\section{References}

1. Jun-Il Yoo, Yonghan Cha, Joonhyeok Kwak, Ha-Yong Kim, Won-Sik Choy. Review on Basicervical Femoral Neck Fracture: Definition, Treatments, and Failures

2. Biomechanical comparison of internal fixation techniques for the treatment of unstable basicervical femoral neck fractures D A Deneka et al. J Orthop Trauma 1997. 
3. Comparative analysis of treatment of basicervical femur fractures in young adults with CCS, DHS, and $\mathrm{PFN}^{\text {is }}$ Anmol Sharma, Anisha Sethi, and Shardaindu Sharma

4. Deneka DA, Simonian PT, Stankewich CJ, Eckert D, Chapman JR, Tencer AF. Biomechanical comparison of internal fixation techniques for the treatment of unstable basicervical femoral neck fractures. J Orthop Trauma. 1997;11(5):337-43.

5. Wilson JN. Injuries of the hip. In: Watson-Jones JN, editor. Fractures and joint injuries. Edinburgh: Churchill Livingstone; 1982;935:957-958.

6. Chen CY, Chiu FY, Chen CM, Huang CK, Chen WM, Chen TH. Surgical treatment of basicervical fractures of femur-a prospective evaluation of 269 patients. J Trauma 2008;64(2):427-9.

7. Kuokkanen HO. Treatment options for basicervical fractures of the femoral neck: a clinical follow-up. Acta Orthop Belg 1991;57(2):162-8.

8. Levy RN, Siegel M, Sedlin ED, Siffert RS. Complications of Ender-pin fixation in basicervical, intertrochanteric, and subtrochanteric fractures of the hip. J Bone Joint Surg Am 1983;65(1):66-9.

9. Massoud EI. Fixation of basicervical and related fractures. Int Orthop. 2010;34(4):577-82.

10. $\mathrm{Su} \mathrm{BW}$, Heyworth BE, Protopsaltis TS, Lipton CB, Sinicropi SM, Chapman CB, et al. Basicervical versus intertrochanteric fractures: an analysis of radiographic and functional outcomes. Orthopedics 2006;29(10):91925 .

11. Watson ST, Schaller TM, Tanner SL, Adams JD, Jeray KJ. Outcomes of low-energy basicervical proximal femoral fractures treated with cephalomedullary fixation. J Bone Joint Surg Am 2016;98(13):1097-102.

12. $\mathrm{Hu} \mathrm{SJ}, \mathrm{Yu} \mathrm{GR}$, Zhang SM. Surgical treatment of basicervical intertrochanteric fractures of the proximal femur with cephalomeduallary hip nails. Orthop Surg. 2013;5(2):124-9.

13. Tasyıkan L, Ugutmen E, Sanel S, Soylemez MS, Ozkan K, Solakoglu C. Short-term results of surgical treatment with cephalomedullary nails for basicervical proximal femoral fractures. Acta Orthop Belg 2015;81(30):427-34. 